\title{
Production and Evaluation of Ag85B:HspX:hFcy1 Immunogenicity as a Recombinant Fc Fusion Multi- Stage Vaccine Candidate Against Mycobacterium Tuberculosis
}

\section{Mohsen Karbalaei}

Jiroft University of Medical Sciences

\section{Arman Mosavat}

Mashhad University of Medical Sciences

\section{Saman Soleimanpour}

Mashhad University of Medical Sciences

Hadi Farsiani

Mashhad University of Medical Sciences

Kiarash Ghazvini

Mashhad University of Medical Sciences

Abbas Ali Amini

Kurdistan University of Medical Sciences

\section{Mojtaba Sankian}

Mashhad University of Medical Sciences

\section{Seyed Abdolrahim Rezaee ( $\sim$ RezaeeR@mums.ac.ir)}

Mashhad University of Medical Sciences, Mashhad, Iran https://orcid.org/0000-0001-6814-5992

\section{Research}

Keywords: Ag85B:HspX:hFcy1 fusion protein, Mycobacterium tuberculosis, Pichia pastoris, subunit vaccine, multi-stage vaccine

Posted Date: October 28th, 2020

DOl: https://doi.org/10.21203/rs.3.rs-96757/v1

License: (1) This work is licensed under a Creative Commons Attribution 4.0 International License. Read Full License 


\section{Abstract}

Aims

Tuberculosis (TB) is one of the life-threatening infectious diseases, caused by Mycobacterium tuberculosis (M.tb). In the present study, a multi-stage M.tb immunodominant Fcy1 fusion protein (Ag85B:HspX:hFcy1) was produced and its immunogenicity as an $\mathrm{hFcyRI}$ targeted delivery systems for selective antigen presentation was evaluated in a mouse model.

Methods and Results

The novel Ag85B:HspX:hFcy1 recombinant fusion protein was designed and expressed in the Pichia pastoris (P. pastoris). After affinity chromatography purification, the purity of Ag85B:HspX:hFcy1 was confirmed by ELISA, SDS-PAGE, and Western blotting methods. The immunogenicity of the construct was evaluated by assessing interferon- $\gamma$ (IFN- $\gamma$ ) and transforming growth factor-beta (TGF- $\beta$ ) in a mouse model. Co-localization results of Ag85B:HspX:hFcy1 with hFcyRI (CD64) confirmed its function for binding with its receptor and inducing Th1 selective responses. There was a significant difference in the expression of both IFN- $\gamma_{1}(P \leq 0.02)$ and $T G F-\beta,(P=0.05)$.

\section{Conclusions}

The co-localization assay confirmed functionally the binding of the Ag85B:HspX:hFcy1 to CD64 (FcyRI). Furthermore, in vitro assay showed that Ag85B:HspX:hFcy1 can stimulate a modulated immune response in favor of anti-intracellular microbes, as IFN- $\gamma$ increased, and also TGF- $\beta$ as an immune-modulatory cytokine prevented the induction of hypersensitivity reactions.

Significance and Impact of Study

The combination of Ag85B as the most immunodominant M.tb Ag with HspX, as an Ag and adjuvant, could open a new venue for more studies for the design of multi-stage subunit vaccines for TB. Of note, an Fcy 1 fusion protein can be considered as a functional approved selective delivery vehicle for targeting antigen-presenting cells (APCs) and inducing cross-presentation.

\section{Introduction}

Tuberculosis (TB) is a re-emerging contagious disease, caused by Mycobacterium tuberculosis (M.tb). Increasing drug-resistant $M$.tb leads to death in the infected patients worldwide, and so named white death (Egedes $\varnothing$ et al. 2020). According to the report of the World Health Organization (WHO) in 2019, nearly 10 million peoples (range, 9.0-11.1 million) were infected with TB, and 1.2 million (among HIV negative) have died worldwide (https://www.who.int/tb/publications/global_report/en/). About two billion peoples are infected with M.tb around the world, but only less than $10 \%$ of them appear active TB; most of the patients possess latent TB and have no clinical symptoms (Cliff et al. 2015). Based on an eligible study, the risk of infection by the active form of TB in the individuals, who co-infected 
simultaneously with HIV and M.tb is $20 \varangle 30$ fold more than those infected with M.tb only (Purmohamad et al. 2020). Unfortunately, the emergence of the multidrug-resistant (MDR) and extensively drug-resistant (XDR) strains to anti-mycobacterial drugs, leads to M.tb infection, which has become the most lethal disease (Mahajan and Dhawale 2015).

Since 1921, Bacille Calmette-Guérin (BCG) vaccine was approved by the WHO and is now used for all populations in epidemic areas. Vaccination by BCG protects infants and children efficiently against meningeal and pulmonary TB (Fatima et al. 2020). However, the protection rate of this vaccine against pulmonary TB in adults is very variable (0ه80\%). BCG is a live attenuated vaccine, and so should not be given to patients with AIDS (Khoshnood et al. 2018). In recent decades, studies have been performed on more effective vaccines than BCG, and some have begun the clinical trial phases. New vaccines are including recombinant BCG (rBCG), DNA vaccines, and subunit vaccines (Kaufmann et al. 2017). Recently, most studies have been conducted on subunit vaccines as new candidates for TB vaccination. In the subunit vaccine structures, one or more immunodominant antigens of microorganisms are used as a fusion construct. Moreover, compared with other new vaccines, these vaccines are more protective than the BCG vaccine and can be used as replacement or booster of the BCG vaccine (Aagaard et al. 2011).

One of the most M.tb immunodominant antigens is the antigen 85 (Ag85) complex. The complex includes three proteins, Ag85A (31 kDa), Ag85B (30 kDa), and Ag85C (31.5 kDa) (Babaki et al. 2017). Members of the Ag85 complex are secretory proteins, and also Ag85A and Ag85B are comprised of approximately $60 \%$ of this complex (Wiker and Harboe 1992). Ag85 complex has a different role during the intracellular pathogenesis of $M$.tb. The fibronectin-binding protein $(f b p)$ encodes Ag85, and bacterium through this antigen binds to fibronectin (Forrellad et al. 2013). Furthermore, Ag85 has a mycolyl transferase activity, which is needed for attachment of mycolic acids to arabinogalactan and the formation of cord factor (Kuo et al. 2012). After the entry of M.tb into the cellular phagosome, the Ag85 complex inhibits the formation of phagolysosome and therefore, has a pivotal role in the intracellular pathogenesis of M.tb (Rens et al. 2018). Consequently, members of the Ag85 complex can promote Th1 cell response to produce interferon-gamma (IFN-y) and interleukin-2 (IL-2) cytokines (Huygen 2014).

BCG vaccine is not able to induce Th1 response in the latent phase of TB and does not protect patients against reactivation and latent form of TB. Another M.tb immunodominant antigen is heat-shock protein $X(H s p X)$, which is expressed in the latent phase of the disease. This protein also named a-crystallin protein (16 kDa), which can induce a strong cellular immune response (Taylor et al. 2012).

The roles of the Fc domain of IgG1 in a fusion construct are the increase of half-life, solubility, and stability of synthetic fusion protein, and also targeted binding to the $\mathrm{Fc}$ gamma receptor (FcyR) on antigen-presenting cells (APCs). After uptake of Fc binding fusion proteins, through $\mathrm{MHC}$ I and II, immunity response of $\mathrm{CD} 4^{+}$and $\mathrm{CD} 8^{+} \mathrm{T}$ cell initiates and consequently leads to the elimination of microorganisms (Levin et al. 2015). 
Considering BCG vaccine inefficacy, finding an efficient TB vaccine candidate is very crucial. In the present study, novel Ag85B:HspX:hFcy1 fusion protein was designed and expressed into the Pichia pastoris (P. pastoris) system, and its immunogenicity was assessed in a mouse model.

\section{Materials And Methods}

\section{Design of Ag85B:HspX:hFcy1 fusion construct}

The DNA fragment of Ag85B:HspX:hFcy1 fusion was 2,048 bp in length, comprising two M.tb genes; fbpB (Ag85B), and $h s p X(\mathrm{HspX})$, bound to each other by a flexible linker, (Gly-Gly-Gly-Gly-Ser) ${ }_{2}$. Next, fusion constructs were bound to the $\mathrm{Fc}$ domain (hinge- $\mathrm{CH} 2-\mathrm{CH} 3$ ) of human $\mathrm{IgG1}$. The sequences of each gene were obtained from the NCBI GenBank database. Restriction sites of Xhol (5') and Notl (3') enzymes were included in-frame with Ag85B-(G4S) ${ }_{2}$-HspX:hFcy1 fusion construct (5' Xhol-Ag85B-(G4S) ${ }_{2}$-HspX:hFcy1Not13'). After final confirmation, this synthetic construct was submitted and registered in the NCBI Nucleotide database. (GenBank accession no.: MK360906; https://www.ncbi.nlm.nih.gov/nuccore/MK360906.1).

\section{In-silico protein modeling of Ag85B:HspX:hFcy1 fusion protein}

Three-dimensional models of the Ag85B:HspX:hFcy1 protein sequences were constructed via homology modeling. Using the basic local alignment search tool (BLAST), sequence homology searches were performed to identify the template proteins by Position-Specific Iterative-BLAST (PSI-BLAST) (http://www.ncbi.nlm.nih.gov/BLAST/). For modeling, the M.tb Ag85B (Protein Data Bank [PDB] entry: 1F0N), heat shock protein 16 (PDB: 3W1Z), and human Fcy1 (PDB entry: 1FC1) were selected as the templates of Ag85B, HspX and Fc domains, respectively. Multiple alignments were carried out on the selected sequences by ClustalX2 (protein weight matrix: BLOSUM series). Model building was performed in the program MODELLER9v20, using a model-ligand algorithm (http://salilab.org/modeller/) (Thompson et al. 1997). Several models at various refinement levels were generated. Three models with the lowest molecular probability density function (molpdf) score were selected for structure refinements. Finally, structure refinements and energy minimization were performed by YASARA Energy Minimization Server and Chimera 1.13 (Krieger et al. 2002; Krieger et al. 2009). The protein dimer form was created by http://galaxy.seoklab.org/. All models were validated, using the program ERRAT at the University of California, Los Angeles (UCLA) (UCLA, http://nihserver.mbi.ucla.edu/saves/) (Colovos and Yeates 1993).

\section{The pPICZaA plasmid as a shuttle vector}

Shuttle vectors can replicate into the different host cells, such as eukaryotic and prokaryotic cells. In this study, pPICZaA plasmid was used with restriction sites of Xhol, Notl, and SaCl and a selective marker gene, sh ble, as a resistant gene, indicating resistance to Zeocin antibiotic.

\section{Codon optimizing of pPICZaA-Ag85B:HspX:hFcy1 recombinant plasmid}


Increasing the expression level needs gene optimization of fusion protein before expression in the target host. Based on the P. pastoris expression system, we used JCat software version 1.0 (Technical University of Braunschweig, USA) for codon optimization. Eventually, a cloned vector, pPICZaA-Ag85B:HspX:hFcy1 was made (Genray, China) along with restriction sites, and quality control of sequencing was certified.

\section{Transformation of the cloned vector into E. coli Top10F'}

Confirmed cloned pPICZaA plasmid was transformed into the Escherichia coli Top10F' (E. coli), as an appropriate host for replication of recombinant plasmid. The transformation was done via the chemical method, using $50 \mathrm{mM}$ calcium chloride $\left(\mathrm{CaCl}_{2}\right)$. Using the Luria Bertani (LB) agar medium (HiMedia, India), containing $25 \mu \mathrm{g} / \mathrm{mL}$ of Zeocin (InvivoGen, USA), the growth of cloned bacteria was evaluated. After $24 \mathrm{~h}$, transformed $E$. coli containing recombinant plasmid (with sh ble gene) were grown on LB agar surface. Subsequently, recombinant pPICZaA plasmids were extracted from bacterial cells via a plasmid extraction kit (Genet Bio, Korea).

\section{Linearizing and cleanup of recombinant plasmids}

Extracted plasmids were linearized with Sacl enzyme (Thermo Scientific, USA). The pPICZaA plasmid has just one excision site for the Sad enzyme. The presence of elution buffer leads to sparking during electroporation and leads to the decrease of the electrocompetent efficacy, resulting in loss of plasmids. Therefore, the cleanup of the plasmid solution was done with the Silica Bead DNA Gel Extraction Kit (Thermo Scientific, USA), and the buffer was replaced by nuclease-free water.

\section{Electroporation of pPICZaA-Ag85B:HspX:hFcy1 using P. pastoris competent cells}

According to the EasySelect Pichia Expression Kit (Invitrogen, USA), competent cells of P. pastoris GS115 (Invitrogen, USA) were prepared by $1 \mathrm{M}$ sorbitol. Through the crossover recombination phenomenon, linear plasmids are integrated into the P. pastoris chromosome. Hence, the Gene Pulser apparatus (BioRad, USA) and electroporator cuvette (green cap) was used. The conditions of electroporation were as follows: 2,000 V, $200 \mathrm{PC}(\mathrm{ohm}), 25 \mathrm{C}(\mu \mathrm{F})$, and $4.4 \mathrm{~ms}$ of time. Transformed yeast cells were cultured in the Yeast Extract-Peptone-Dextrose-Sorbitol (YPDS) agar medium, containing $100 \mu \mathrm{g} / \mathrm{mL}$ of Zeocin. After incubating plates for $2 \varangle 4$ days in an incubator at $29^{\circ} \mathrm{C}$, Zeocin resistant clones were grown.

\section{Selection of the best transformant clones and colony-PCR}

Based on the EasySelect Pichia expression manual, confirmation of integrated plasmid into the $P$. pastoris chromosome was done with universal primers, alcohol oxidase (AOX1) and a-factor. The length of the PCR product of the gene of interest with $A O X 1$ primers ( $5^{\prime}$ and $\left.3^{\prime}\right)$ was 2,636 bp, and with a-factor (5') and $A O X 1$ (3') was 2,347 bp. Also, grown clones for evaluation of the high crossover recombination were cultured on YPDS agar plates with different concentration of Zeocin, including 200,500, 1,000, and $2,000 \mu \mathrm{g} / \mathrm{mL}$ of Zeocin. After $48 \mathrm{~h}$, recombinant clones, growing in high levels of Zeocin (e.g., 1,000 or 
$2,000 \mu \mathrm{g} / \mathrm{mL}$ ) were selected as the best recombinant clones (high-copy number integrated clones) for expression of recombinant protein in a large scale.

\section{Optimization and large scale protein expression}

In the present study, methanol concentrations, incubation times, and culture medium container were optimized. For replication and production of biomass, at first, selective clones (three clones) were cultured into $50 \mathrm{~mL}$ of buffered minimal glycerol medium (BMGY) in a baffled flask in a shaker incubator at $29^{\circ} \mathrm{C}$ and $220 \mathrm{rpm}$, for $18 \otimes 24 \mathrm{~h}$, until the $\mathrm{OD}_{600}$ of 2-6 was reached. To prevent the adhesion of secretory recombinant protein to the interior wall of the flask expanded roller bottles (Grainer, Germany) were used instead of non-siliconized baffled flasks. The roller bottle of $2,500 \mathrm{~mL}$ volume was filled with $250 \mathrm{~mL}$ of sterile buffered minimal methanol medium (BMMY). The induction of protein expression under different methanol concentrations is an important factor in the Pichia expression system. The primary concentration of methanol for inducing protein expression was $0.5 \%$. The optimum methanol concentration for high-level expression of recombinant protein was $2 \%$, but the expression was not observed in $4 \%$ of methanol. The maximum level of protein expression occurred on the $7^{\text {th }}$ day of methanol induction time.

\section{Purification of Ag85B:HspX:hFcy1 recombinant protein}

According to the secretory nature of Ag85B:HspX:hFcy1 fusion protein, the supernatant of the BMMY medium was centrifuged and collected. Briefly, using $1 \mathrm{M}$ sodium phosphate buffer, the $\mathrm{pH}$ of the supernatant was adjusted to 7 (neutral $\mathrm{pH}$ ), and then filtrated by cellulose acetate (CA) $0.45 \mu \mathrm{M}$ membrane filter (filtraTECH, France). Purification of protein was done by the HiTrap rProtein A Sepharose Fast Flow column (GE Healthcare, USA). At first, by using $20 \mathrm{mM}$ sodium phosphate buffer $\mathrm{pH}$ 7, citrate elution buffer $\mathrm{pH} 3$, and then again, sodium phosphate buffer, the column was regenerated. The supernatant was passed into the column (flow rate, $3 \mathrm{~mL} / \mathrm{min}$ ), and finally, recombinant protein was eluted by $100 \mathrm{mM}$ glycine buffer $\mathrm{pH}$ 3. Immediately, the $\mathrm{pH}$ of the eluted protein was adjusted to 7 by $1 \mathrm{M}$ Tris/HCl buffer $\mathrm{pH}$ 9. Using Vivaspin 20 ultrafiltration spin filter (Sartorius Stedim, Germany), the protein solution was concentrated, and also glycine buffer was replaced by phosphate-buffered saline (PBS).

\section{Analysis of Ag85B:HspX:hFcy1 protein by SDS-PAGE and Western blotting}

To perform SDS-PAGE, and Western blot analyses, two gels containing $12 \%$ polyacrylamide were prepared. One gel was used for SDS-PAGE, and then protein bands were stained with Coomassie Brilliant Blue G-250 (Merck, Germany), using the Bio-Rad Mini PROTEAN II apparatus (BIO-RAD, USA). Another gel was used for Western blotting, and confirmation of recombinant protein was done by the goat antihuman IgG-HRP antibody (Santa Cruz, USA).

\section{Co-localization of Ag85B:HspX:hFcy1 fusion protein}


Binding of Ag85B:HspX:hFcy1 recombinant protein to human Fcy receptor I (FcyRI) on macrophage/monocyte as APCs, was confirmed by direct immunofluorescence assay. Shortly, $5 \mathrm{~mL}$ of monocytes of venous blood were separated by sterile Lympholyte-H solution (CEDARLANE, Canada). After washing by PBS and purification of monocytes, using a fixator (ethanol/acetone), monocytes were fixed on a slide in a $-20^{\circ} \mathrm{C}$ freezer. The slide was stained with PE anti-human CD64 (FcyRI) (BioLegend, USA), and incubated at $37^{\circ} \mathrm{C}$ in a humid chamber for $1 \mathrm{~h}$. The slide was washed and again incubated with Ag85B:HspX:hFcy1 protein for $1 \mathrm{~h}$. Finally, the slide was washed and stained with goat anti-human IgG Fc-FITC (BioLegend, USA), and mounted with $90 \%$ glycerin and nail polish. Stained monocytes were visualized by a fluorescence microscope (Nikon Eclipse E200, Japan) with 100X magnification, and images were captured.

\section{Preparation of Ag85B:HspX:hFcy1 subunit vaccine}

The appropriate concentration of the new subunit vaccine was determined, using the bicinchoninic acid (BCA) method. Based on the universal protocol for injection of TB vaccine to mice, $50 \mu \mathrm{g} / \mathrm{mL}$ of recombinant protein in PBS was suitable for injection to mouse. For the best induction of cell-mediated immune (CMI) response, adjuvants dimethyl-dioctadecyl ammonium bromide (DDA) (Sigma-Aldrich, USA), and trehalose-6,6-dibehenate (TDB) (Avanti Polar Lipids; Merck, Germany) were used, in the ratio of 5 to 1, respectively (Henriksen-Lacey et al. 2010). The Ag85B:HspX:hFcy1 recombinant protein was mixed with DDA/TDB complex just $1 \mathrm{~h}$ before injection to the mice.

\section{Immunization of C57BL/6 mice by Ag85B:HspX:hFcy1 subunit vaccine}

Evaluation of Ag85B:HspX:hFcy1 recombinant protein immunogenicity was performed, using 6₫8 weeks pathogen-free female C57BL/6 mice, purchased from the Pasteur Institute of Iran (Tehran, Iran). In brief, the mice ( $n=42)$ were divided into six groups. Each group includes seven mice: (a) the group receiving 100 $\mu \mathrm{L}$ of sterile PBS; (b) the group receiving $50 \mu \mathrm{L}$ of BCG vaccine; (3) the group receiving $100 \mu \mathrm{L}$ of Ag85B:HspX:hFcy1 (50 $\mu \mathrm{g} / \mathrm{mL})$ plus $100 \mu \mathrm{L}$ of DDA/TDB; (d) the group receiving $100 \mu \mathrm{L}$ of $50 \mu \mathrm{g} / \mathrm{mL}$ of Intravenous Immunoglobulin (IVIg) plus $100 \mu \mathrm{L}$ of DDA/TDB; (e) the group receiving primed BCG, and then boosted by Ag85B:HspX:hFcy1 plus DDA/TDB; (f) the group receiving BCG and boosted by IVIg, and DDA/TDB. The groups of 1 and 2 were injected subcutaneously just one time, on the $1^{\text {st }}$ day, but other groups were immunized on days $1^{\text {st }}, 14^{\text {th }}, 28^{\text {th }}$, and $42^{\text {nd }}$. Finally, the mice were dissected, and their spleen lymphocytes (splenocytes) were seeded in triplicate and incubated in a plate for $10 \otimes 12 \mathrm{~h}$ at $37^{\circ} \mathrm{C}$ in a $\mathrm{CO}_{2}$ incubator. Then, cultured cells were collected and centrifuged in $1.5 \mathrm{~mL}$ microtubes. Supernatants were harvested, then, Wizol reagent (Wizbiosolutions, Korea) was added on cell pellets. After that mRNA was extracted, using the High Pure RNA Isolation Kit (Roche, Germany), according to the manufacturers' protocol and kept at $-70^{\circ} \mathrm{C}$ until further processing.

\section{Evaluation of cytokine gene expression by qRT-PCR}

First, cDNA molecules were synthesized, using a cDNA kit (Thermo Scientific) according to manufacturers' instructions. Finally, cDNA (double-stranded DNA) was amplified by quantitative real-time 
PCR technique, and a Rotorgen Q-6000 real-time PCR machine (Qiagen, Germany). The expression of transforming growth factor-beta $(T G F-\beta)$ and IFN- $\gamma$ genes was assessed via the EvaGreen real-time PCR (Thermo Scientific, USA). The primer sequences used for TGF- $\beta$,IFN- $\gamma$, and beta-2 microglobulin $(\beta 2 M)$ genes are shown in Table 1.

Table 1. Primers sequences of IFN- $\gamma, T G F-\beta$, and $\beta 2 M$.

\begin{tabular}{|llll|}
\hline $\begin{array}{l}\text { Target } \\
\text { gene }\end{array}$ & Forward $\mathbf{( 5}^{\prime} \mathbf{3}^{\prime} \mathbf{)}$ & Reverse $\mathbf{( 5}^{\prime} \mathbf{3}^{\prime} \mathbf{)}$ & $\begin{array}{l}\text { Amplicon size } \\
\text { (bp) }\end{array}$ \\
\hline $\boldsymbol{I F N} \boldsymbol{- \boldsymbol { y }}$ & TGGGTTCTCTTGGCTGTTACTG & GAGTTCCATTATCCGCTACATCTG & 105 \\
\hline $\boldsymbol{T G F - \boldsymbol { \beta }}$ & GCAAGTGGACATCAACGGGTT & CGCACGCAGCAGTTCTTCTC & 192 \\
\hline $\boldsymbol{\beta 2} \boldsymbol{M}$ & CTTGTCTTTCAGCAAGGACTGG & CCACTTAACTATCTTGGGCTGTG & 127 \\
\hline
\end{tabular}

IFN-ץ. Interferon-gamma; TGF- $\beta$. Transforming growth factor-beta; $\beta 2 M$ : Beta 2 microglobulin.

\section{Statistical analysis}

The data were analyzed, using SPSS Version 16 (SPSS, Chicago, IL, USA), and $p<0.05$ was considered statistically significant. To evaluate the gene expression levels in the mice studied group, the KruskalWallis test was performed.

\section{Results}

\section{Design of Ag85B:HspX:hFcy1 fusion construct}

Firstly, the reference sequences of $f b p B, h s p X$, and human Fc IgG1 genes were obtained from the NCBI database. The sequences were fused from $\mathrm{N}$-terminus to $\mathrm{C}$-terminus, with flexible linker $\left(\mathrm{G}_{4} \mathrm{~S}\right)_{2}$, and the addition of Xho1, and Notl restriction sites. Sub-cloning of Ag85B:HspX:hFcy1 fusion construct was performed into the pPICZaA plasmid. The insertion site of Ag85B:HspX:hFcy1 construct was between the $5^{\prime} A O X 1$ and $3^{\prime} A O X 1$ domains in the plasmid. The Ag85B:HspX:hFcy1 fusion protein (dimer conformation) was expressed in the P. pastoris system. The schematic illustrations of Ag85B:HspX:hFcy1 construct and dimeric form of Ag85B:HspX:hFcy1 fusion protein were represented in Figs 1 and 2.

\section{Molecular modeling of Ag85B:HspX:hFcy1 recombinant fusion protein}

In the present study, 20 models were created using Modeler software; three models with the lowest molpdf were selected for structure refinements and the energy was minimized by YASARA Energy Minimization Server and Chimera 1.13. Among refined models, the most valid model achieved 94.55 ERRAT scores (from 0-100). Considering Fc properties in forming a dimer, our model is designated as a 
dimer, which was created by http://galaxy.seoklab.org/. A solid ribbon model of Ag85B:HspX:hFcy1 fusion protein is depicted in Fig. 3.

\section{Colony-PCR of selected transformant clones}

As noted, following the electroporation of the Ag85B:HspX:hFcy1 DNA fragment into the P. pastoris chromosome, insertion of the construct was confirmed by $A O X 1$ and a-factor primers, using PCR amplification. The length of Ag85B:HspX:hFcy1 DNA fragment was 2,048 bp. Based on the crossover recombination mechanism, using $5^{\prime} A O X 1$ and $3^{\prime} A O X 1$ primers, the primary DNA size of Ag85B:HspX:hFcy 1 was summed with 588 bp, but using 5'a-factor and 3'AOX1 primers, the primary DNA size of Ag85B:HspX:hFcy1 was added to 299 bp (Fig. 4).

\section{SDS-PAGE and Western blot analyses of Ag85B:HspX:hFcy1 fusion protein}

Following purification of Ag85B:HspX:hFcy1 fusion protein by HiTrap rProtein A Sepharose Fast Flow Column, the neutralization performed by Tris/ $\mathrm{HCl}, \mathrm{pH}$ 7. The protein was identified by SDS-PAGE and confirmed via Western blotting. Based on information obtained from the ExPASy database, the molecular weight of Ag85B:HspX:hFcy1 recombinant protein was $77 \mathrm{kDa}$ with a pl of 5.92, consisting of 709 amino acids (Fig. 5).

\section{Methanol concentration for optimum induction of expression}

The primary methanol concentration for induction of protein expression in P. pastoris system is $0.5 \%$ of BMMY volume $(\mathrm{v} / \mathrm{v})$. The maximum level of expression for all proteins is $2 \varangle 2.5 \%(\mathrm{v} / \mathrm{v})$ of methanol (Wang et al. 2010). Usually, expressing cells can bear the concentration of methanol up to $5 \%$, but concentrations more than that are very toxic and inhibit the production of proteins (Shi et al. 2003). In this study, two methanol concentrations, $2 \%$ and $4 \%$, were applied. The results of SDS-PAGE showed that optimum expression of Ag85B:HspX:hFcy1 protein was obtained on 2\% methanol concentration (Fig. 6)

\section{APC-targeting of human monocytes by immunofluorescence assay}

Binding of Ag85B:HspX:hFcy1 recombinant fusion protein to FcyRI (CD64) of human monocyte/macrophages was confirmed, using immunofluorescence assay. Consequently, PE mouse antihuman CD64 (red) and goat anti-human IgG Fc-FITC (green) bind to CD64 and Fc domain of Ag85B:HspX:hFcy1 recombinant fusion protein, respectively. The immunofluorescence microscope images showed that the inside and surfaces of the cells were emitted red and green signals, respectively (Fig. 7).

\section{The IFN- $\gamma$ and TGF- $\beta$ expression levels}

The results of the Kruskal-Wallis test showed that there is a significant difference in the IFN- $y$ expression $(P \leq 0.02)$. The highest transcription level with a mean of 1.35 was shown in Ag85B:HspX:hFcy $1+\mathrm{BCG}$ group, which had been stimulated by PHA. On the other hand, the expression level with a mean of 0.03 
was shown in the IVIg group, which had been stimulated by IVIg. The IVlg plays an immune modulator role (as an anti-inflammatory), so that following subcutaneous injection in mice, it causes an increase in the FcyRIIB expression on the surface of mouse macrophage cells, resulting in the inactivation of macrophages. The IFN- $y$ expression levels are shown in Fig. 8.

The results of the Kruskal-Wallis test revealed that there is a significant difference in the TGF- $\beta$ expression $(P=0.05)$. Like IFN- $\gamma$, the highest expression level with a mean of 0.24 was shown in the Ag85B:HspX:hFcy1 + BCG group, which had been stimulated by PHA. On the other hand, the lowest expression level with a mean of 0.004 was shown in IVIg + BCG group, stimulated by IVIg. The TGF- $\beta$ expression levels are shown in Fig. 9.

\section{Discussion}

Historically, regarding the lethal nature of M.tb and the emergence of new drug-resistant strains (e.g., MDR and XDR strains), TB treatment has encountered a serious challenge. On the other hand, the BCG vaccine has disadvantages such as low efficiency in adult pulmonary TB, $(0 \otimes 80 \%)$ inefficiency, latent TB, and HIV positive subjects (Farsiani et al. 2016; Kebriaei et al. 2016; Mosavat et al. 2016). Nowadays, subunit vaccines that are produced, comprising recombinant single or fusion proteins can be regarded as an appropriate alternative for the BCG vaccine (Babaki et al. 2019).

The M.tb infections are found in both active ( $10 \otimes 20 \%$ of cases) and latent ( $80 \otimes 90 \%$ of cases) forms; therefore, multistage subunit vaccines are the best candidates for TB infection, because they are created from both active and latent M.tb antigens (Farsiani et al. 2016; Kebriaei et al. 2016; Mosavat et al. 2016). For producing an effective subunit vaccine, there are two main strategies: firstly, using a targeted delivery system to target APCs for selective presentation to the Th0 cells, in order to induce appropriate immune responses; and secondly, utilizing an appropriate adjuvant in multi injection and as a multi booster to potentiate immune responses. The main powerful APCs such as DCs encode the FcyR1 (CD64), which can bind to the Fc fragment of human IgG1 or mouse lgG2a and induce cross-presentation phenomenon (Keler et al. 2000; Adamova et al. 2005; Rawool et al. 2008).

Although, covalently bonded protein to the Fc fragment has various advantages, including an increase of half-life, solubility, and stability of Fc-bound proteins, and increase of quality and quantity of purification (Czajkowsky et al. 2012). The main focus of our study was the selective targeting of DCs for the induction of Th1 and the production of proper cellular immunity. In addition, for inducing appropriate responses, Ag85B:HspX:hFcy1 recombinant protein was mixed with TDB and DDA as Th1 adjuvants (Agger et al. 2008). Moreover, why Ag85 was chosen, because M.tb as an intracellular parasitic organism can secret many different virulence factors such as, ESAT-6 (EsXA), CFP-10 (EsXB), PPEs and Ag85B, to intervene with the host responses and survive (Forrellad et al. 2013; Tang et al. 2014).

In our previous M.tb-host study in TB-positive and TB-negative patients, the gene expression assessments showed that the main virulence factor of M.tb was Ag85B and the complex of ESAT-6-CFP-10 that M.tb uses to combat host responses. On the other hand, the effective host's response to eliminate M.tb was a 
Th1 cellular immune response, mainly against the predominant M.tb antigen (Ag85), implicating in the polarization of the response by the expression of transcription factor (T-bet), modulatory factors (IDO and FoxP3) and activation of effector macrophages, activating oxygen-dependent pathway, iNOS and proteolytic activities (Sharebiani et al. 2020). Therefore, Ag85 was used as the most virulence factor for activation and dissemination of M.tb, while it is the most immunodominant Ag for host immune responses to produce protective immunity.

TB is an immunopathology disease of type IV hypersensitivity and the appropriate host response is a potent Th1 along with a moderate TGF- $\beta$ (Soleimanpour et al. 2015; Babaki et al. 2019). As a result, T-bet induces Th1 immune response to produce IFN- $\gamma$ and IL-2, and Foxp3 induces Treg to secrete TGF- $\beta$ and modulate the immune response to M.tb in a proper degree. The Th1 cells and their cytokines activated oxygen-dependent, proteolytic and autophagy of infected macrophages to eliminate M.tb (Mullen et al. 2001; Bhatt and Salgame 2007; Pitt et al. 2013). However, M.tb-HspX was used as the main expressed antigen in the latent phase of the disease and has also adjuvant activities (Lew et al. 2020). Using these two Mt.b immunogens that covalently bind to Fc fragment (Ag85B:HspX:hFcy1) may form an immunodominant complex to induce Th1 proper immune response, which can be effective as a vaccine and also as a therapy in altering latency for activation and exacerbation of TB.

In recent years, attempts for making subunit vaccines have dedicated a large proportion of non-cellular vaccines. Several useful subunit vaccine candidates have been constructed by the use of Ag85, and some well-known are Hybrid 1 (Ag85B-ESAT-6 vaccine), HyVac4 (Ag85B-TB10.4 vaccine) and Hybrid 56 (Ag85B- ESAT-6- Rv2660) vaccines (Babaki et al. 2017). Given that, in our previous studies mouse Fcy2aM.tb fusion recombinant proteins were used, showing acceptable results (Soleimanpour et al. 2015; Farsiani et al. 2016; Mosavat et al. 2016; Babaki et al. 2019); therefore, based on those results and more search in the literature, it seems that this complex would be more effective.

The findings of this study showed that when dimerized Fcy fragment bound to FcyRI, it functionally induced Th1 and cross-presentation (Fig. 7), which consequently led to the increased IFN- $y$ expression (Junker et al. 2020). Focusing on a high concentration of IFN-y alone for making a protective immune response against $M$.tb infection could not give promising results. TB is immunopathological damage to type IV hypersensitivity reactions, so that for producing protective immunity, the specific stimulator must induce precise immuno-modulatory responses.

In the present study, the presentation of TGF- $\beta$ was also increased. TGF- $\beta$ inhibited the excessive response of Th1 pro-inflammatory cytokines such as IFN- $\gamma$ and prevented inflammatory reactions (Harris et al. 2007). Thus, Th1 imbalance can significantly promote the development and progression of TB (Reljic et al. 2009). As the present study findings showed, although the level of TGF- $\beta$ increased, it was not comparable with IFN- $y$ and may modulate pro-inflammatory cytokines, for making such precise appropriate immuno-protective responses. Furthermore, preventing TB flare-up as an immunopathological disease requires a modulated Th1 immune response with dominated IFN- $\gamma$ and TGF- $\beta$ moderate secretion. 
The importance of recombinant Fc-fusion proteins is inevitably dependent on their biophysical, biochemical and pharmacological characteristics, which exist in the constant region of the Fc fragment. Many medications with the FDA approved products are available for many different ranges of medical intervention (Czajkowsky et al. 2012; Soleimanpour et al. 2017). Furthermore, attempts for making appropriate vaccines against infectious diseases such as Ebola (Konduru et al. 2011), TB (Soleimanpour et al. 2015; Farsiani et al. 2016; Mosavat et al. 2016), Influenza (Loureiro et al. 2011) and SARS-CoV-2 (Ren et al. 2020) are still ongoing.

This study has some limitations; one limitation of utilizing FcyRs antigen-targeting is the potential interaction with the inhibitory FcyR (FcyR IIB) on APCs, which could lead to a tolerogenic immune response. Co-localization of yeast made Fc fusion protein and FcRs could aid in better understanding the type of immune response. Utilizing a suitable animal model such as a mouse challenge model is needed to assess the protective immune response of Ag85B:HspX:hFcy1 against TB.

In conclusion, the co-localization experiments showed the proper binding of the present Fc fusion protein to $\mathrm{CD} 64$ (FcyRI) which in turn, confirms the suitable folding and functionally active protein for inducing Th1 responses. Furthermore, consistent with this finding, in vitro assay showed that Ag85B:HspX:hFcy1 was able to stimulate a modulated immune response in favor of anti-intracellular microbes, as IFN- $\gamma$ was increased, as well as TGF- $\beta$ as an immune-modulatory cytokine, which can prevent the induction of hypersensitivity reactions. From our previous studies focusing on making immunogene M.tb fusion, taken together, it seems to us that Ag85B as the most immunodominant Ag in binding to HspX as an M.tb $\mathrm{Ag}$ and active adjuvant may provide a new venue for more attempts in making subunit multi-stage vaccine for TB. Of note, Fcy 1 fusion protein can be considered as a functional approved selective delivery vehicle for targeting APCs and inducing cross-presentation.

\section{Declarations}

\section{ETHICS APPROVAL}

The animal experiments were reviewed, and approved by the Institutional Animal Care and Use Committee of Mashhad University of Medical Sciences (IR.MUMS.REC. 951414) and has been performed according to National Institutes of Health guideline (NIH publication No. 85-23, revised 1985). During the experiments, the vaccinated mice were monitored every day.

\section{CONSENT FOR PUBLICATION}

Not applicable.

\section{AVAILABILITY OF DATA AND MATERIALS}

The datasets generated and/or analyzed during the current study are included in this paper and available from the corresponding author (S.A.R. Rezaee) upon reasonable request. 
CONFLICT OF INTEREST

The authors declare that they have no conflicts of interest regarding this manuscript.

\section{FUNDING}

This study was financially supported by the Vice-Chancellor for Research and Technology, Mashhad University of Medical Sciences, under Grant [MUMS 951414].

\section{AUTHORS' CONTRIBUTIONS}

Doing experiments and manuscript drafting: MK, AM, SS and HF; In Silico protein modeling: AA; Research advisors: SS, HF, MS and AM; Research director, Conception and design of the study, Data analysis: SAR and KG. All authors have read and approved the final manuscript.

\section{ACKNOWLEDGMENTS}

This research was subjected to PhD dissertation in Medical Bacteriology by Mohsen Karbalei. The authors have great thanks to the Vice-Chancellor for Research and Technology, Mashhad University of Medical Sciences (MUMS) for financially supporting of the present study.

\section{References}

Aagaard, C., Hoang, T., Dietrich, J., Cardona, P.-J., Izzo, A., Dolganov, G., Schoolnik, G.K., Cassidy, J.P., Billeskov, R. and Andersen, P. (2011) A multistage tuberculosis vaccine that confers efficient protection before and after exposure. Nature medicine17, 189.

Adamova, E., Walsh, M.C., Gosselin, D.R., Hale, K., Preissler, M.T., Graziano, R.F. and Gosselin, E.J. (2005) Enhanced antigen-specific antibody and cytokine responses when targeting antigen to human FcGAMMA receptor type I using an anti-human FcGAMMA receptor type I-streptavidin fusion protein in an adjuvantfree system. Immunological investigations34, 417-429.

Agger, E.M., Rosenkrands, I., Hansen, J., Brahimi, K., Vandahl, B.S., Aagaard, C., Werninghaus, K., Kirschning, C., Lang, R., Christensen, D., Theisen, M., Follmann, F. and Andersen, P. (2008) Cationic liposomes formulated with synthetic mycobacterial cordfactor (CAF01): a versatile adjuvant for vaccines with different immunological requirements. PLoS One3, e3116.

Babaki, M.K.Z., Soleimanpour, S. and Rezaee, S.A. (2017) Antigen 85 complex as a powerful Mycobacterium tuberculosis immunogene: biology, immune-pathogenicity, applications in diagnosis, and vaccine design. Microbial Pathogenesis112, 20-29.

Babaki, M.K.Z., Taghiabadi, M., Soleimanpour, S., Moghadam, M.S., Mosavat, A., Amini, A.A., Mohammadi, A. and Rezaee, S.A. (2019) Mycobacterium tuberculosis Ag85b: hfcy1 recombinant fusion protein as a selective receptor-dependent delivery system for antigen presentation. Microbial pathogenesis129, 68-73. 
Bhatt, K. and Salgame, P. (2007) Host innate immune response to Mycobacterium tuberculosis. J Clin Immuno/27, 347-362.

Cliff, J.M., Kaufmann, S.H., McShane, H., van Helden, P. and O'Garra, A. (2015) The human immune response to tuberculosis and its treatment: a view from the blood. Immunol Rev264, 88-102.

Colovos, C. and Yeates, T.O. (1993) Verification of protein structures: patterns of nonbonded atomic interactions. Protein Sci2, 1511-1519.

Czajkowsky, D.M., Hu, J., Shao, Z. and Pleass, R.J. (2012) Fc-fusion proteins: new developments and future perspectives. EMBO molecular medicine4, 1015-1028.

Egedes $\varnothing$, P.J., Hansen, C.W. and Jensen, P.S. (2020) Preventing the White Death: Tuberculosis Dispensaries. The Economic Journa/130, 1288-1316.

Farsiani, H., Mosavat, A., Soleimanpour, S., Sadeghian, H., Akbari Eydgahi, M.R., Ghazvini, K., Sankian, M., Aryan, E., Jamehdar, S.A. and Rezaee, S.A. (2016) Fc-based delivery system enhances immunogenicity of a tuberculosis subunit vaccine candidate consisting of the ESAT-6:CFP-10 complex. Mol Biosyst12, 21892201.

Fatima, S., Kumari, A., Das, G. and Dwivedi, V.P. (2020) Tuberculosis vaccine: A journey from BCG to present. Life Sci252, 117594.

Forrellad, M.A., Klepp, L.I., Gioffre, A., Sabio y Garcia, J., Morbidoni, H.R., de la Paz Santangelo, M., Cataldi, A.A. and Bigi, F. (2013) Virulence factors of the Mycobacterium tuberculosis complex. Virulence4, 3-66.

Harris, J., De Haro, S.A., Master, S.S., Keane, J., Roberts, E.A., Delgado, M. and Deretic, V. (2007) T helper 2 cytokines inhibit autophagic control of intracellular Mycobacterium tuberculosis. Immunity27, 505-517.

Henriksen-Lacey, M., Christensen, D., Bramwell, V.W., Lindenstrøm, T., Agger, E.M., Andersen, P. and Perrie, Y. (2010) Liposomal cationic charge and antigen adsorption are important properties for the efficient deposition of antigen at the injection site and ability of the vaccine to induce a CMI response. $J$ Control Release145, 102-108.

Huygen, K. (2014) The Immunodominant T-Cell Epitopes of the Mycolyl-Transferases of the Antigen 85 Complex of M. tuberculosis. Front Immuno/5, 321.

Junker, F., Gordon, J. and Qureshi, O. (2020) Fc Gamma Receptors and Their Role in Antigen Uptake, Presentation, and T Cell Activation. Front Immuno/11, 1393.

Kaufmann, S.H., Weiner, J. and von Reyn, C.F. (2017) Novel approaches to tuberculosis vaccine development. Int J Infect Dis56, 263-267. 
Kebriaei, A., Derakhshan, M., Meshkat, Z., Eidgahi, M.R., Rezaee, S.A., Farsiani, H., Mosavat, A., Soleimanpour, S. and Ghazvini, K. (2016) Construction and immunogenicity of a new Fc-based subunit vaccine candidate against Mycobacterium tuberculosis. Mol Biol Rep43, 911-922.

Keler, T., Guyre, P.M., Vitale, L.A., Sundarapandiyan, K., van De Winkel, J.G., Deo, Y.M. and Graziano, R.F. (2000) Targeting weak antigens to CD64 elicits potent humoral responses in human CD64 transgenic mice. J Immuno/165, 6738-6742.

Khoshnood, S., Heidary, M., Haeili, M., Drancourt, M., Darban-Sarokhalil, D., Nasiri, M.J. and Lohrasbi, V. (2018) Novel vaccine candidates against Mycobacterium tuberculosis. Int J Biol Macromo/120, 180-188.

Konduru, K., Bradfute, S.B., Jacques, J., Manangeeswaran, M., Nakamura, S., Morshed, S., Wood, S.C., Bavari, S. and Kaplan, G.G. (2011) Ebola virus glycoprotein Fc fusion protein confers protection against lethal challenge in vaccinated mice. Vaccine29, 2968-2977.

Krieger, E., Joo, K., Lee, J., Lee, J., Raman, S., Thompson, J., Tyka, M., Baker, D. and Karplus, K. (2009) Improving physical realism, stereochemistry, and side-chain accuracy in homology modeling: four approaches that performed well in CASP8. Proteins: Structure, Function, and Bioinformatics77, 114-122.

Krieger, E., Koraimann, G. and Vriend, G. (2002) Increasing the precision of comparative models with YASARA NOVA-a self-parameterizing force field. Proteins: Structure, Function, and Bioinformatics47, 393-402.

Kuo, C.J., Bell, H., Hsieh, C.L., Ptak, C.P. and Chang, Y.F. (2012) Novel mycobacteria antigen 85 complex binding motif on fibronectin. J Biol Chem287, 1892-1902.

Levin, D., Golding, B., Strome, S.E. and Sauna, Z.E. (2015) Fc fusion as a platform technology: potential for modulating immunogenicity. Trends in biotechnology33, 27-34.

Lew, M.H., Norazmi, M.N. and Tye, G.J. (2020) Enhancement of immune response against Mycobacterium tuberculosis HspX antigen by incorporation of combined molecular adjuvant (CASAC). Mol Immuno/117, 54-64.

Loureiro, S., Ren, J., Phapugrangkul, P., Colaco, C.A., Bailey, C.R., Shelton, H., Molesti, E., Temperton, N.J., Barclay, W.S. and Jones, I.M. (2011) Adjuvant-free immunization with hemagglutinin-Fc fusion proteins as an approach to influenza vaccines. J Viro/85, 3010-3014.

Mahajan, N.S. and Dhawale, S. (2015) Linked pyridinyl-thiadiazoles: Design and synthesis as potential candidate for treatment of XDR and MDR tuberculosis. European journal of medicinal chemistry102, 243248.

Mosavat, A., Soleimanpour, S., Farsiani, H., Sadeghian, H., Ghazvini, K., Sankian, M., Jamehdar, S.A. and Rezaee, S.A. (2016) Fused Mycobacterium tuberculosis multi-stage immunogens with an Fc-delivery 
system as a promising approach for the development of a tuberculosis vaccine. Infection, Genetics and Evolution39, 163-172.

Mullen, A.C., High, F.A., Hutchins, A.S., Lee, H.W., Villarino, A.V., Livingston, D.M., Kung, A.L., Cereb, N., Yao, T.-P. and Yang, S.Y. (2001) Role of T-bet in commitment of TH1 cells before IL-12-dependent selection. Science292, 1907-1910.

Pitt, J.M., Blankley, S., McShane, H. and O'Garra, A. (2013) Vaccination against tuberculosis: how can we better BCG? Microbial pathogenesis58, 2-16.

Purmohamad, A., Azimi, T., Nasiri, M.J., Goudarzi, M., Zangiabadian, M., Sedighian, H. and Fooladi, A.A.I. (2020) HIV-Tuberculous Meningitis Co-infection: A Systematic Review and Meta-analysis. Curr Pharm Biotechnol.

Rawool, D.B., Bitsaktsis, C., Li, Y., Gosselin, D.R., Lin, Y., Kurkure, N.V., Metzger, D.W. and Gosselin, E.J. (2008) Utilization of Fc receptors as a mucosal vaccine strategy against an intracellular bacterium, Francisella tularensis. The Journal of Immunology180, 5548-5557.

Reljic, R., Paul, M.J. and Arias, M.A. (2009) Cytokine therapy of tuberculosis at the crossroads. Expert Rev Respir Med3, 53-66.

Ren, W., Sun, H., Gao, G.F., Chen, J., Sun, S., Zhao, R., Gao, G., Hu, Y., Zhao, G., Chen, Y., Jin, X., Fang, F., Chen, J., Wang, Q., Gong, S., Gao, W., Sun, Y., Su, J., He, A., Cheng, X., Li, M., Xia, C., Li, M. and Sun, L. (2020) Recombinant SARS-CoV-2 spike S1-Fc fusion protein induced high levels of neutralizing responses in nonhuman primates. Vaccine38, 5653-5658.

Rens, C., Laval, F., Wattiez, R., Lefèvre, P., Dufrasne, F., Daffé, M. and Fontaine, V. (2018) I3-Ag85 effect on phthiodiolone dimycocerosate synthesis. Tuberculosis108, 93-95.

Sharebiani, H., Hajimiri, S., Abbasnia, S., Soleimanpour, S., Asnaashari, H., Valizadeh, N., Derakhshan, M., Pilpa, R., Firouzeh, A. and Ghazvini, K. (2020) Game Theory Applications in Host-Microbe Interactions: Mycobacterium tuberculosis infection as an example. bioRxiv, 2019.2012. 2026.888602.

Shi, X., Karkut, T., Chamankhah, M., Alting-Mees, M., Hemmingsen, S.M. and Hegedus, D. (2003) Optimal conditions for the expression of a single-chain antibody (scFv) gene in Pichia pastoris. Protein Expr Purif28, 321-330.

Soleimanpour, S., Farsiani, H., Mosavat, A., Ghazvini, K., Eydgahi, M.R., Sankian, M., Sadeghian, H., Meshkat, Z. and Rezaee, S.A. (2015) APC targeting enhances immunogenicity of a novel multistage Fcfusion tuberculosis vaccine in mice. Appl Microbiol Biotechno/99, 10467-10480.

Soleimanpour, S., Hassannia, T., Motiee, M., Amini, A.A. and Rezaee, S.A. (2017) Fcy1 fragment of IgG1 as a powerful affinity tag in recombinant Fc-fusion proteins: immunological, biochemical and therapeutic properties. Crit Rev Biotechno/37, 371-392. 
Tang, X.L., Zhou, Y.X., Wu, S.M., Pan, Q., Xia, B. and Zhang, X.L. (2014) CFP10 and ESAT6 aptamers as effective Mycobacterial antigen diagnostic reagents. J Infect69, 569-580.

Taylor, J.L., Wieczorek, A., Keyser, A.R., Grover, A., Flinkstrom, R., Karls, R.K., Bielefeldt-Ohmann, H., Dobos, K.M. and Izzo, A.A. (2012) HspX-mediated protection against tuberculosis depends on its chaperoning of a mycobacterial molecule. Immunology and cell biology $90,945-954$.

Thompson, J.D., Gibson, T.J., Plewniak, F., Jeanmougin, F. and Higgins, D.G. (1997) The CLUSTAL_X windows interface: flexible strategies for multiple sequence alignment aided by quality analysis tools. Nucleic Acids Res25, 4876-4882.

Wang, Z., Wang, Y., Zhang, D., Li, J., Hua, Z., Du, G. and Chen, J. (2010) Enhancement of cell viability and alkaline polygalacturonate lyase production by sorbitol co-feeding with methanol in Pichia pastoris fermentation. Bioresour Techno/101, 1318-1323.

Wiker, H.G. and Harboe, M. (1992) The antigen 85 complex: a major secretion product of Mycobacterium tuberculosis. Microbiol Rev56, 648-661.

\section{Figures}

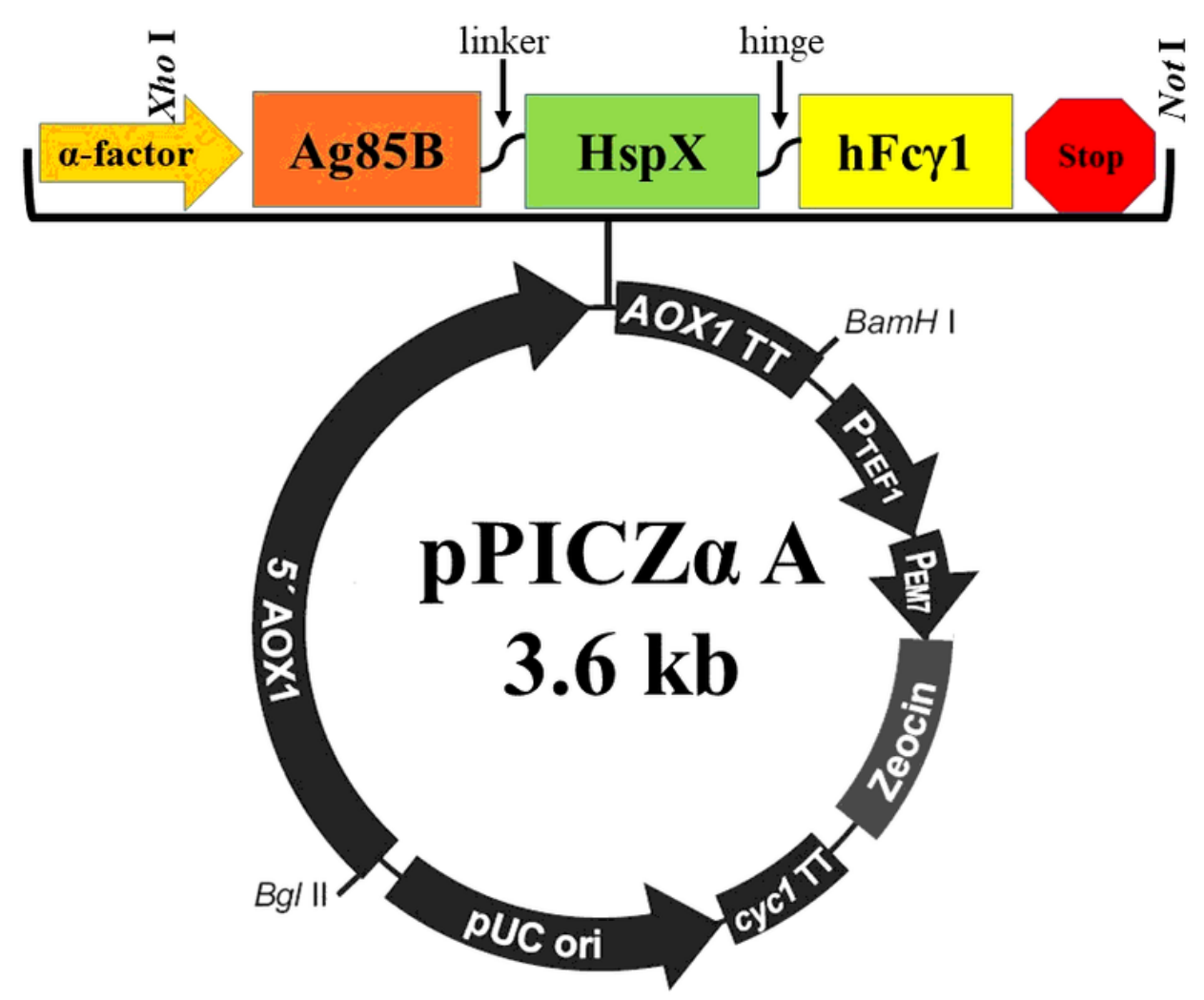

Figure 1 
Schematic illustration of Ag85B:HspX:hFcy1 fusion construct. Primary design of Fc fusion recombinant protein with Xhol and Notl enzymes restriction sites and position of its subcloning in the pPICZaA plasmid. Ag85: antigen 85B; HspX: heat shock protein X; hFcy1: human Fc gamma receptor 1; Linker: (Gly4Ser)2.

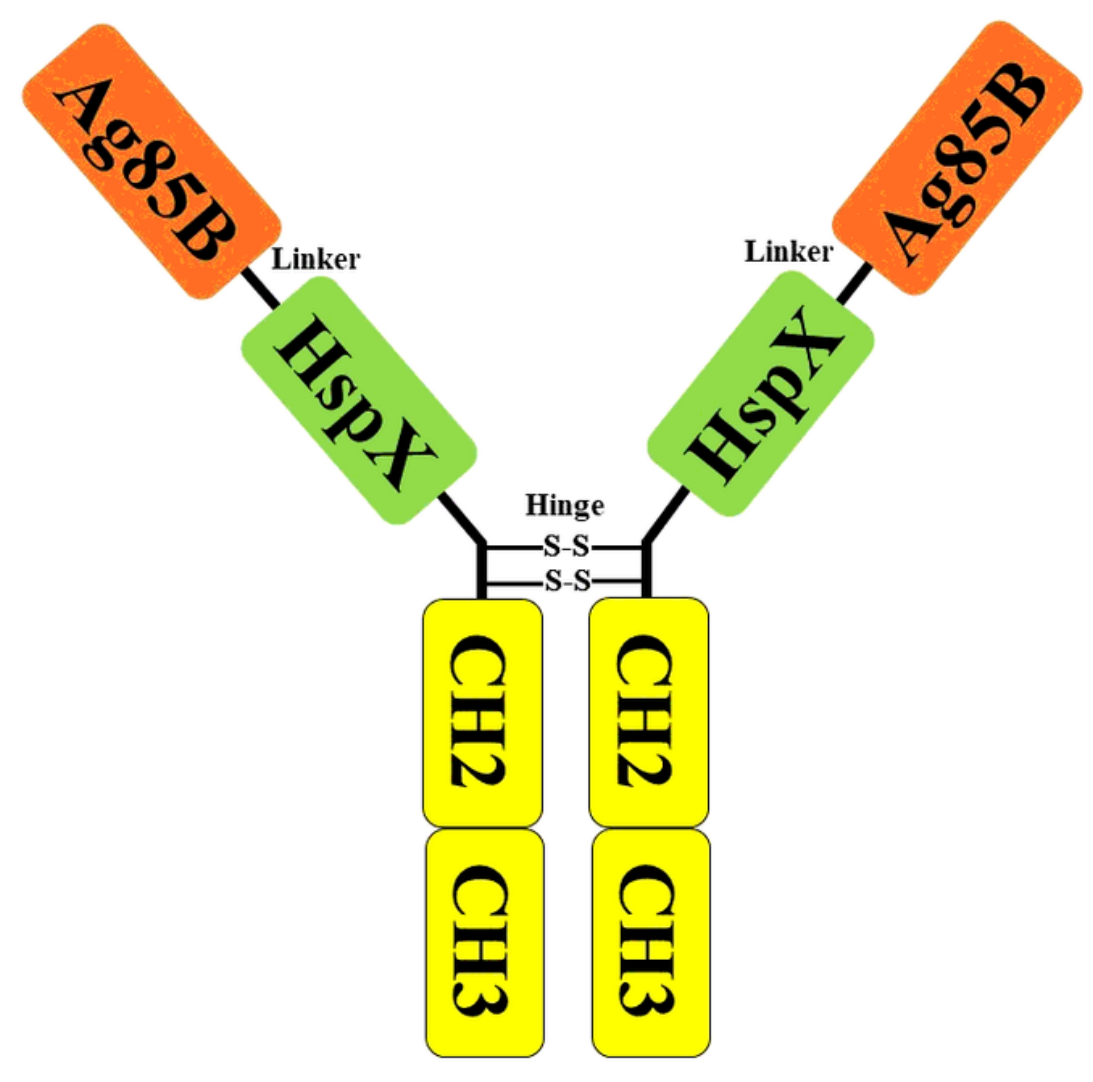

Figure 2

Dimeric form of Ag85B:Hsp:hFcy1 fusion protein. Ag85: antigen 85B; HspX: heat shock protein X; hFcy1: human Fc gamma receptor 1; Linker: (Gly4Ser)2. 


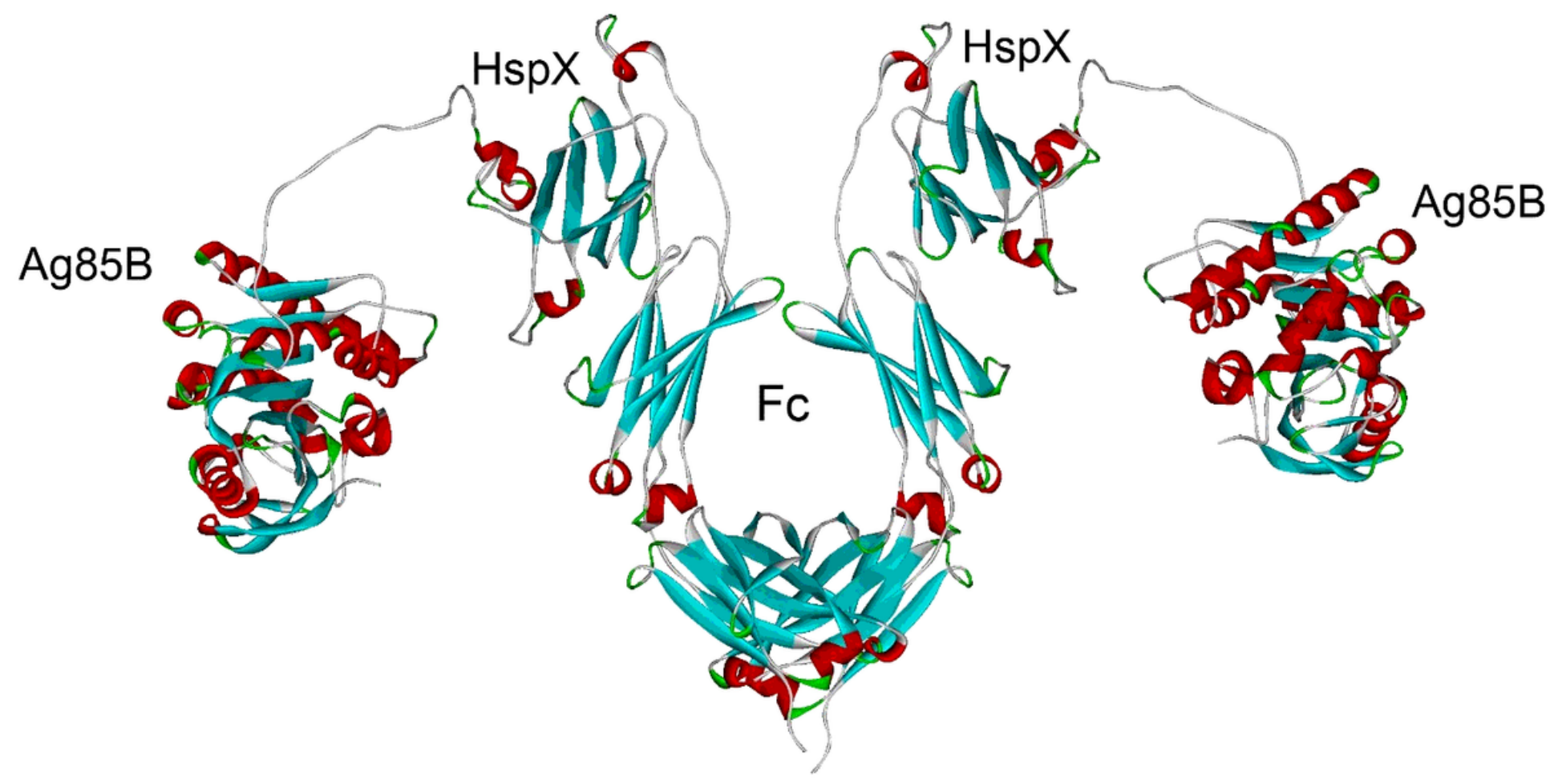

Figure 3

Molecular modeling of Ag85B:HspX:hFcy1 fusion protein. Ag85B:HspX:hFcy1 recombinant protein was modelled by MODELLER9v20. Energy minimization performed by YASARA Energy Minimization Server and Chimera 1.13 and validated by programs ERRAT in which the best final model achieved 94.55 score. The protein dimer form created by http://galaxy.seoklab.org/. Graph was created using Accelrys DS Visualizer v2.1. Ag85: antigen 85B; HspX: heat shock protein X; hFcy1: human Fc gamma receptor 1. 


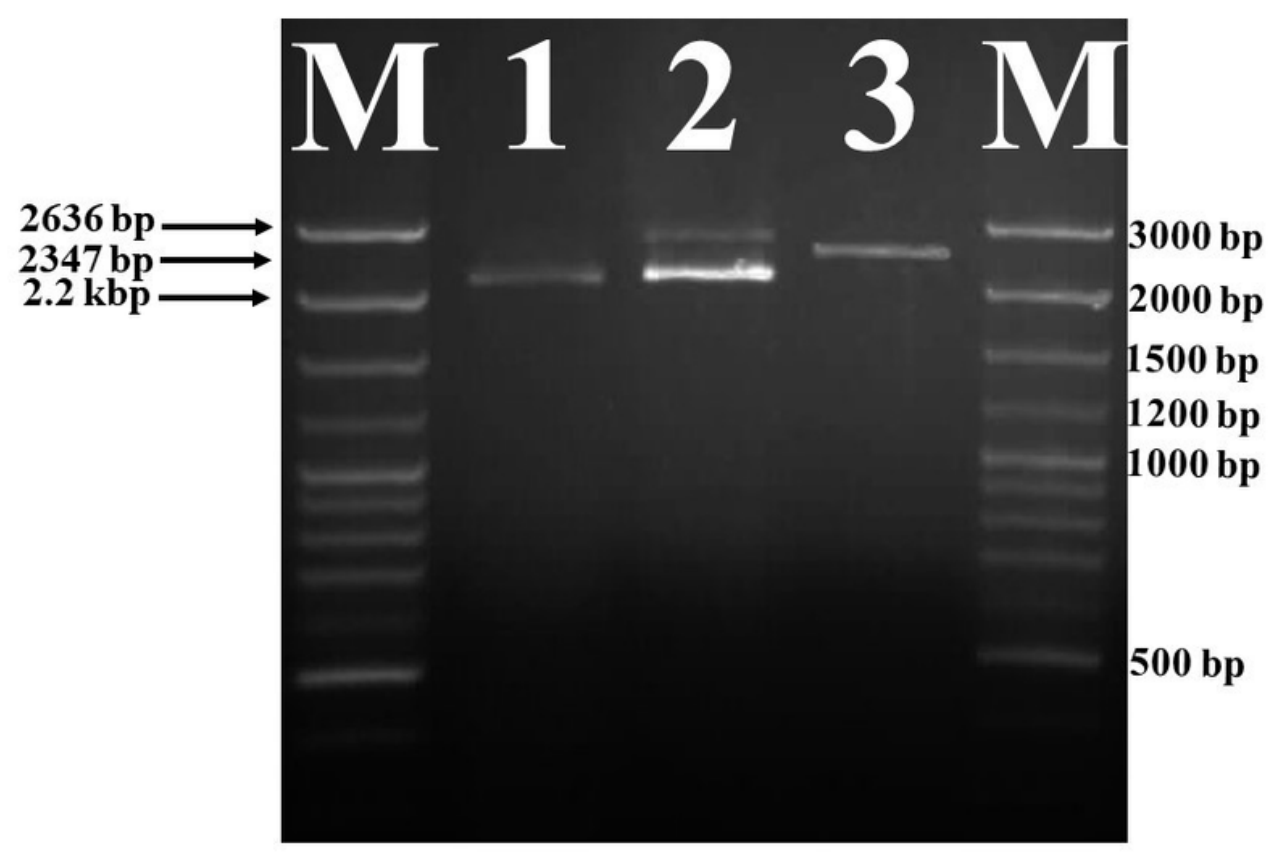

Figure 4

Confirmation of cloned DNA fragment of Ag85B:HspX:hFcy1 fusion by PCR. Lane M, 100 bp plus DNA marker; Lane 1, P. pastoris hose keeping gene ( $2.2 \mathrm{kp})$ as internal control by use of 5'AOX1 and 3'AOX1 primers; Lane 2, our interest DNA fragment plus $588(2,048+588=2,636 \mathrm{bp})$ and internal control $(\sim 2.2$ kp) by use of 5'AOX1 and 3'AOX1 primers; Lane 3, Ag85B:HspX:hFcy1 DNA fragment plus $299(2,048+$ $299=2,347$ bp) by use of 5'a-factor and 3'AOX1 primers. Ag85: antigen 85B; HspX: heat shock protein X; hFcy1: human Fc gamma receptor 1; AOX1: alcohol oxidase. 


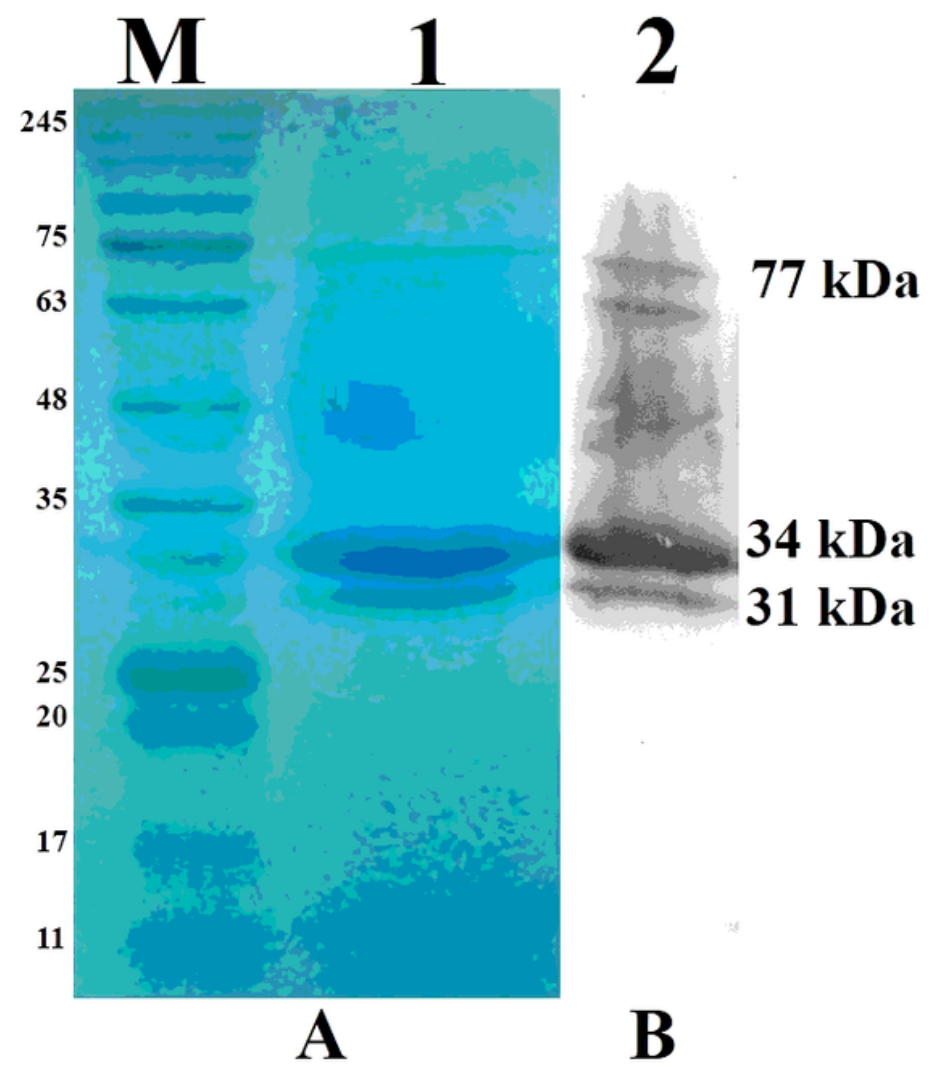

\section{Figure 5}

Identification and confirmation of Ag85B:HspX:hFcy1 protein by SDS-PAGE, and Western blotting. (A) 12\% SDS-PAGE analysis of the purified protein Ag85B:HspX:hFcy1: Lane M, protein marker (kDa); Lane 1, Ag85B:HspX:hFcy1 recombinant protein. (B) Western blotting analysis of the purified protein: Lane 2, confirmation of recombinant protein by the goat anti-human IgG-HRP antibody. Ag85: antigen 85B; HspX: heat shock protein $\mathrm{X} ; \mathrm{hFcy} 1$ : human Fc gamma receptor 1. 


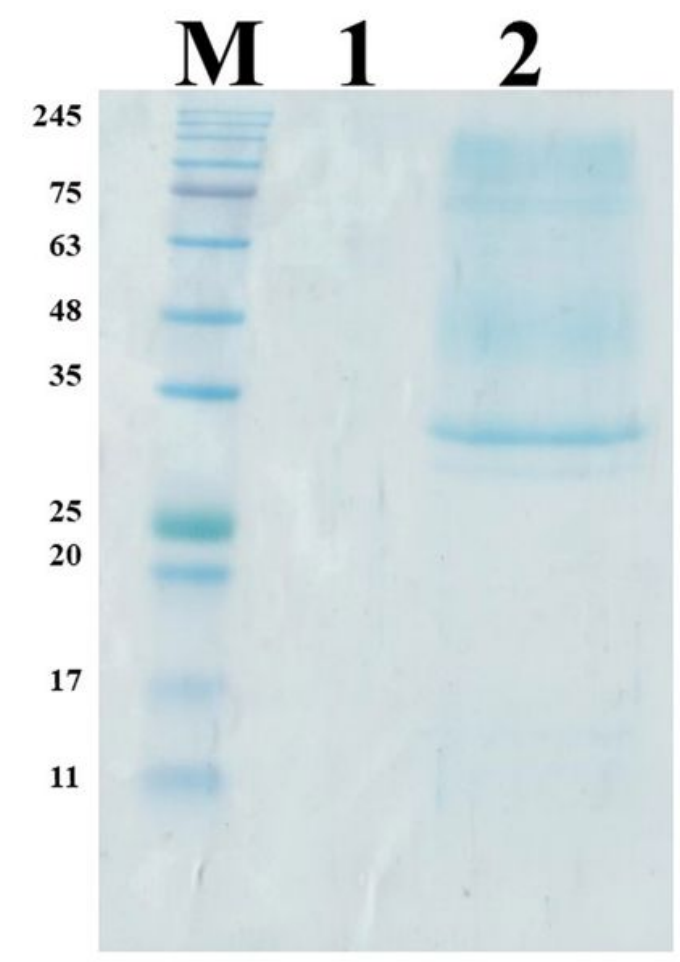

\section{Figure 6}

Optimum and non-toxic concentration of methanol for Ag85B:HspX:hFcy1 expression. Lane M, protein marker $(\mathrm{kDa})$; Lane 1, usage of $4 \%$ methanol concentration in BMMY medium and very low level expression of protein, so that no bands were seen on SDS-PAGE gel; Lane 2, usage of $2 \%$ methanol concentration with maximum level expression of protein in BMMY medium. Ag85: antigen 85B; HspX: heat shock protein $\mathrm{X} ; \mathrm{hFcy} 1$ : human Fc gamma receptor 1. 

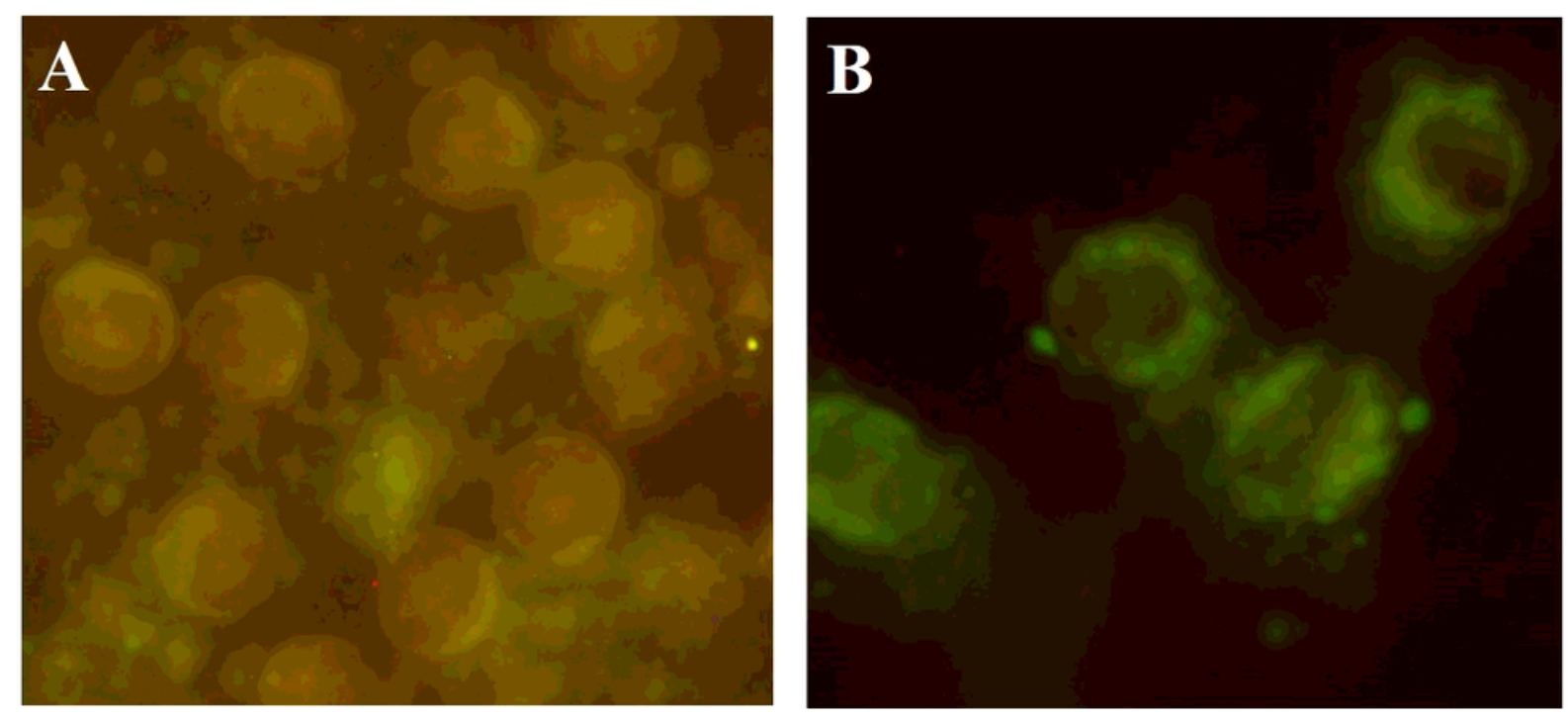

\section{Figure 7}

Co-localization of Ag85B:HspX:hFcy1 protein by immunofluorescence assay. (A) Human monocytes were stained only by PE mouse anti-human CD64 (red), and were seen with red signal. (B) Human monocytes which bonded to Ag85B:HspX:hFcy1 protein were stained by both immunofluorescence colors, PE mouse anti-human CD64 (red) and goat anti-human IgG Fc-FITC (green); insides, and surfaces of the cells were seen with red and green signal, respectively. Ag85: antigen 85B; HspX: heat shock protein X; hFcy1: human Fc gamma receptor 1. 


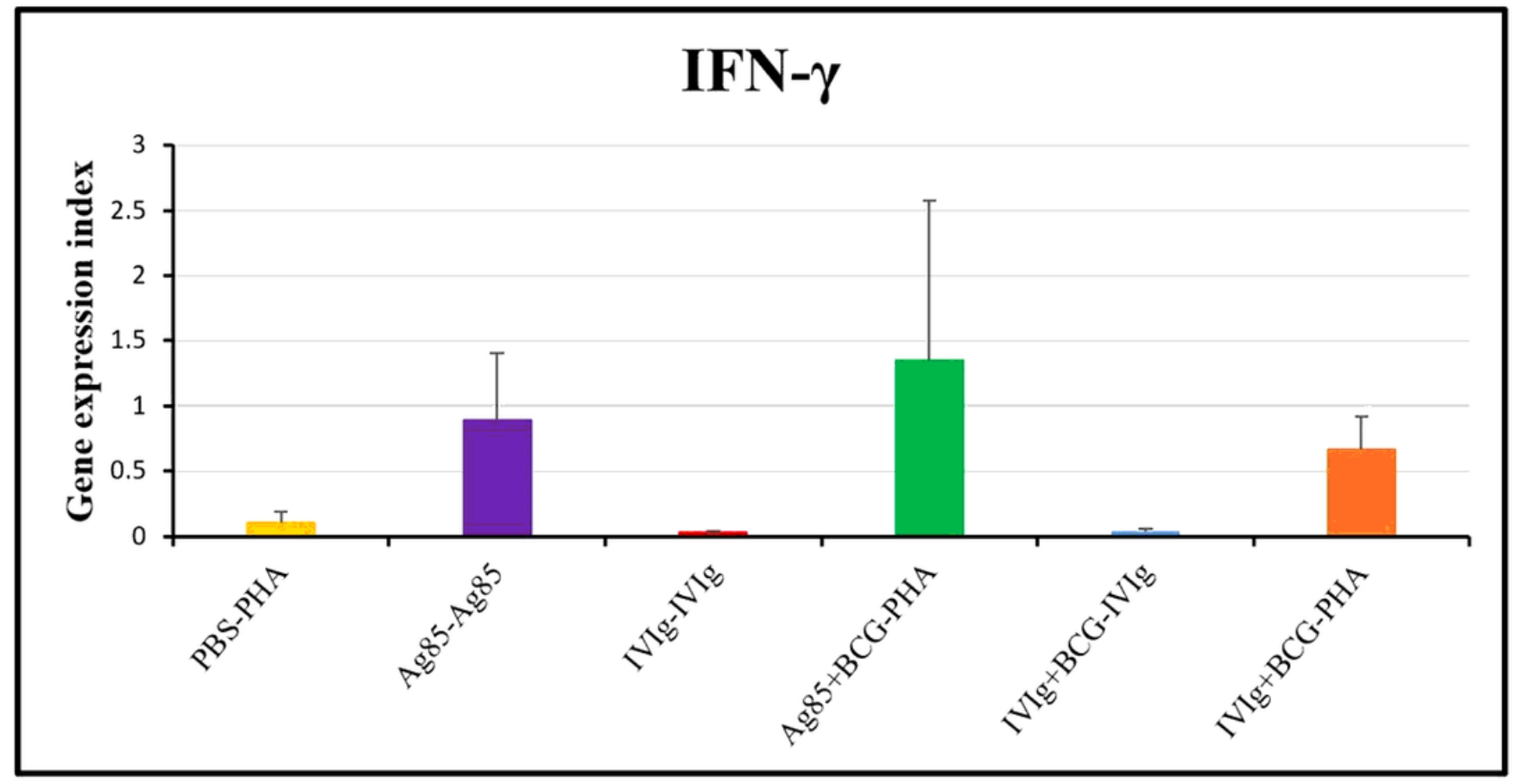

\section{Figure 8}

The IFN-y expression levels index in the mice studied groups. $\neg$ The highest expression level was observed in Ag85B:HspX:hFcy1 + BCG group (green bar) and vs, the lowest expression level was related to IVIg group (red bar). The words have been separated by a dash; word(s) in the left of dash: injected substance(s), and word(s) in the right of dash: stimulating substance(s). Ag85: antigen 85B; HspX: heat shock protein X; hFcy1: human Fc gamma receptor 1; IVIg: Intravenous Immunoglobulin. 


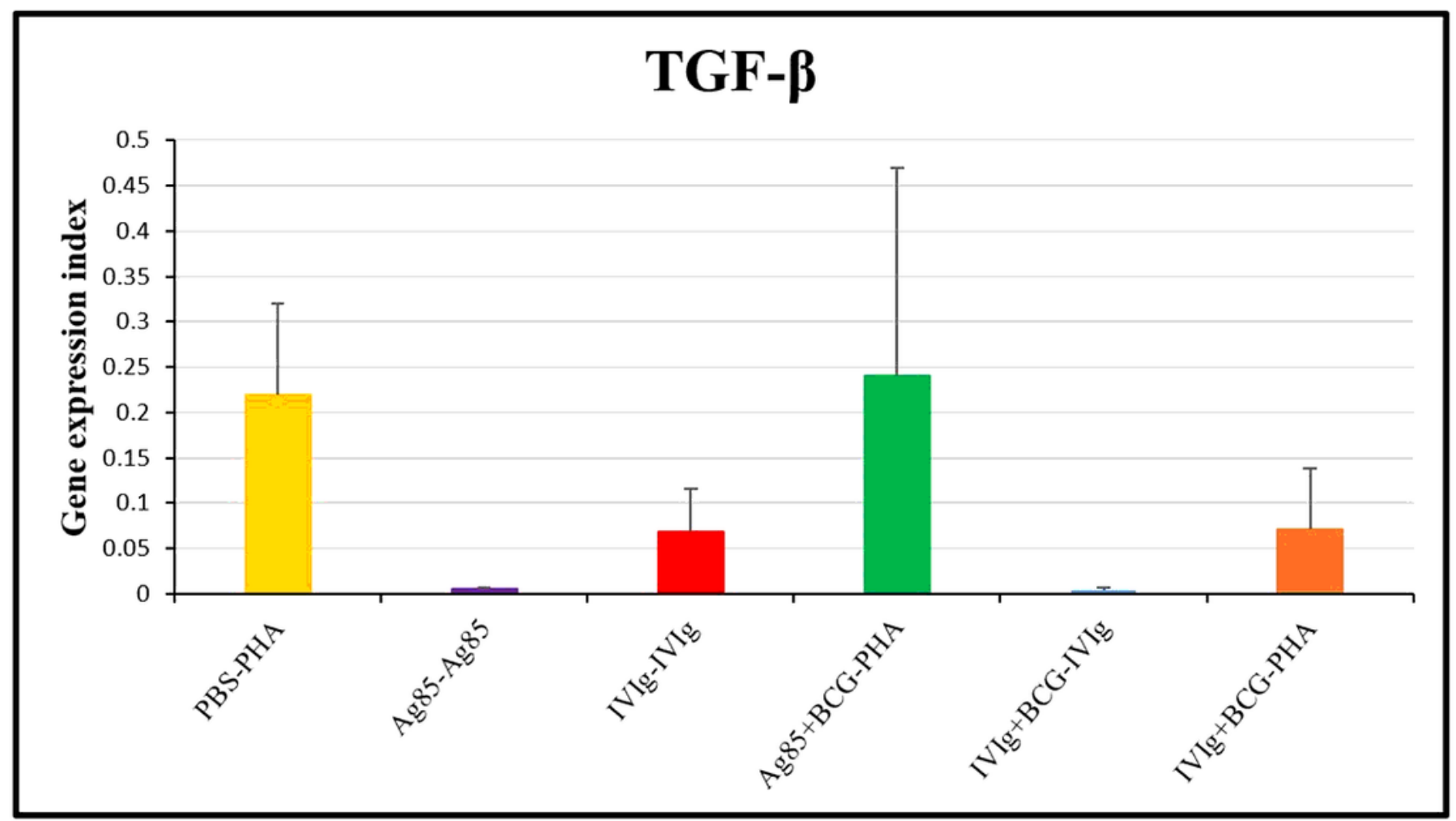

Figure 9

The TGF- $\beta$ expression levels index in the mice studied groups. $\neg$ The highest expression level was observed in Ag85B:HspX:hFcy1 + BCG group (green bar) and vs, the lowest expression level is related to IVIg + BCG group (blue bar). The words have been separated by a dash; word(s) in the left of dash: injected substance(s), and word(s) in the right of dash: stimulating substance(s). Ag85: antigen 85B; HspX: heat shock protein X; hFcy1: human Fc gamma receptor 1; IVIg: Intravenous Immunoglobulin. 